IS-5135

UC-606

\title{
X-ray K-edge Analysis of Drain Lines in Wilhelm Hall, Ames Laboratory
}

Terry Jensen and Craig Whitmore

Ames Laboratory and Center for NDE

Iowa State University

Ames, IA 50011

January 5, 1999

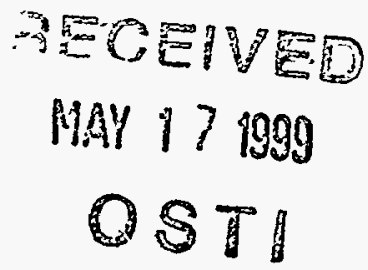

\section{ABSTRACT}

From August 12-27, $1998 \mathrm{X}$-ray K-edge measurements were made on drain lines in seven rooms in Wilhelm Hall, Ames Laboratory. The purpose of these measurements was to determine the extent of thorium (and other heavy metal) contamination inside these pipes. The $\mathrm{K}$-edge method is a noninvasive inspection technique that can provide accurate quantification of heavy metal contamination interior to an object.

Of the seven drain lines inspected, one was found to have no significant contamination, three showed significant thorium deposits, two showed mercury contamination, and one line was found to contain mercury, thorium and uranium. The $\mathrm{K}$-edge measurements were found to be consistent with readings from hand-held survey meters, and provided much greater detail on the location and amount of heavy metal contamination.

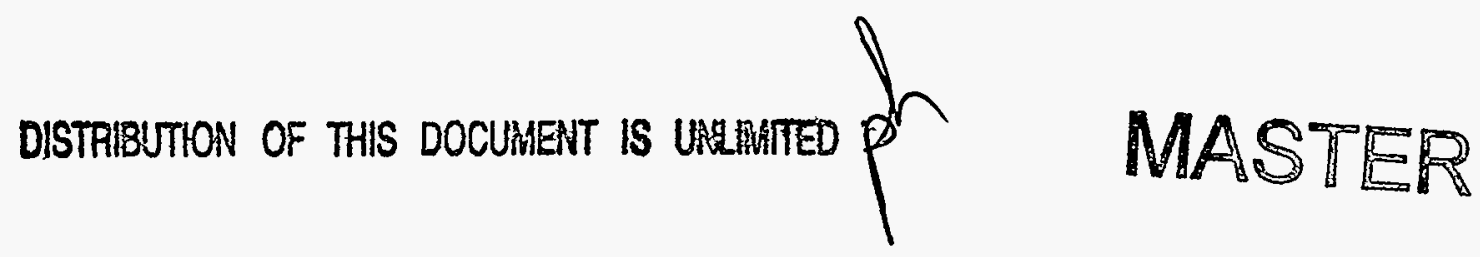




\section{DISCLAIMER}

This report was prepared as an account of work sponsored by an agency of the United States Government. Neither the United States Government nor any agency thereof, nor any of their employees, makes any warranty, express or implied, or assumes any legal liability or responsibility for the accuracy, completeness, or usefulness of any information, apparatus, product, or process disclosed, or represents that its use would not infringe privately owned rights. Reference herein to any specific commercial product, process, or service by trade name, trademark, manufacturer, or otherwise does not necessarily constitute or imply its endorsement, recommendation, or favoring by the United States Government or any agency thereof. The views and opinions of authors expressed herein do not necessarily state or reflect those of the United States Government or any agency thereof. 


\section{DISCLAIMER}

Portions of this document may be illegible in electronic image products. Images are produced from the best available original document. 


\section{INTRODUCTION}

From 1949 to 1953 the U.S. Government conducted research activities on thorium-232 in a building located on the Iowa State University campus, which is now called Harley Wilhelm Hall, and is under the control of Ames Laboratory, U.S. DOE. As a result of these activities various areas of this building were contaminated. Since cessation of thorium research activities in Wilhelm Hall, several surveys of the building have been performed and mitigation activities have been taken where feasible. ${ }^{1}$ During several remodeling projects, much of the contamination was removed or sealed in place. Remaining contamination is confined to several well-identified areas in the building. Although this remaining contamination does not represent a hazard for normal day-to-day activities in the building, it presents risks that must be controlled whenever any modifications are made to the building, or when maintenance is done on some of the building infrastructure.

One area of contamination is in many of the sink traps and drain lines in the building. Some pipes have been removed and disposed of as radioactive waste when contamination was found. (In some instances mercury was also found in the pipes.) Based on these examples it appears that contamination is tied up in scale inside the pipes, predominantly in traps and low spots. Measurements made external to the pipes using portable radiation survey meters indicate significant contamination in several locations. However, quantitative estimates of contamination are difficult to obtain due to the unknown amount of scale inside the pipes, and the fact that the pipes are wrapped in asbestos insulation.

To obtain improved measurements of contamination inside these pipes it was decided to apply the X-ray K-edge inspection technology ${ }^{2,3}$ developed at Ames Laboratory. This technology is based on the measurement of the energy spectrum of an X-ray beam transmitted through the sample. The spectrum will display sharp transitions at characteristic energies (the K-edge energy) corresponding to the presence of heavy metals (see Fig. 1). The energy at which the step occurs identifies the element, and the change in X-ray transmission on either side of the edge can be used to calculate the amount of that element present. The measurement precision is determined by the statistics on either side of the K-edge. Typical precision of $10 \%$ can be achieved within a few minutes. Further details on the K-edge technique can be found in Ref. 2. 


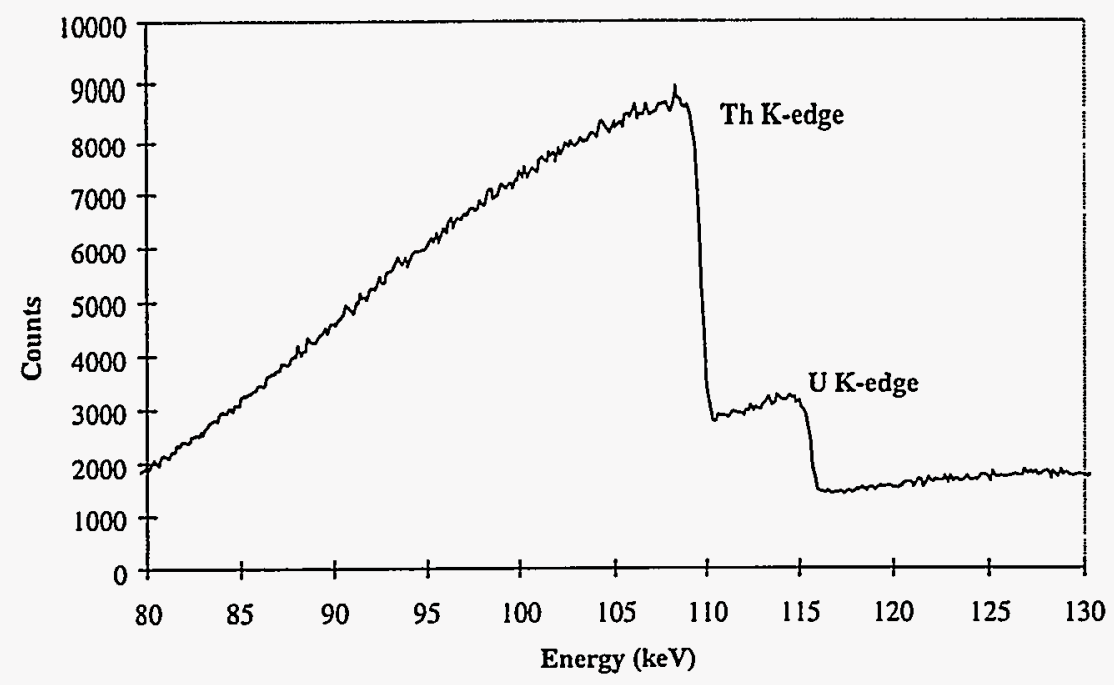

Figure 1. Example spectrum from $\mathrm{K}$-edge inspection of thorium and uranium calibration foils through $1 / 8$ inch of steel.

\section{METHOD}

The prototype K-edge detector consists of an X-ray source and an energy-sensitive detector interfaced to a personal computer. The X-ray source is an industrial X-ray tube, and the detector is a high purity Ge crystal. Both source and detector are collimated to define a narrow ( $<1 \mathrm{~mm}$ dia.) beam used to probe the sample. For inspections in Wilhelm Hall, the source and detector were mounted on a mechanical lift so they could be positioned around a drain line as indicated in Fig. 2. The drain lines are 3-4 inch diameter pipes wrapped with insulation. For each of the drain lines a series of $\mathrm{K}$-edge measurements were made to map out any contamination. A grid was laid out on a plastic sheet and attached to each section of pipe as a reference. The layout of the first two floors of Wilhelm Hall is presented in Appendix A, with the rooms inspected using the $\mathrm{K}$-edge technique indicated.

Initial results were available online to indicate the level of contamination and serve as a guide for determining the frequency of measurements for each pipe. Due to the known history of activities in Wilhelm Hall, the focus was on thorium. At each point on a trap data were collected until the statistical precision of the thorium measurement was at least $10 \%$ or until 10 minutes had passed. Examples of observed spectra are shown in Figs. 3 and 4. These were two of the more striking examples of contamination found.

Radiographs of the drain traps were also taken. These were of mixed quality due to the constraints in locating the film and X-ray tube, but generally gave a feel for the location of scale and other deposits in the traps. Most traps showed quite a variation in X-ray absorption, with some having wires or large pieces of absorbing material embedded in the 
scale. Because these images do not reproduce well on paper, they are not presented in this report. Rather, a summary of observations for each pipe is given.

Measurements were also made along each drain line using a hand-held survey meter. This indicated the level of radioactivity in the pipes but gave no information on what elements were present. This measurement also cannot detect the presence of nonradioactive heavy metals such as mercury.

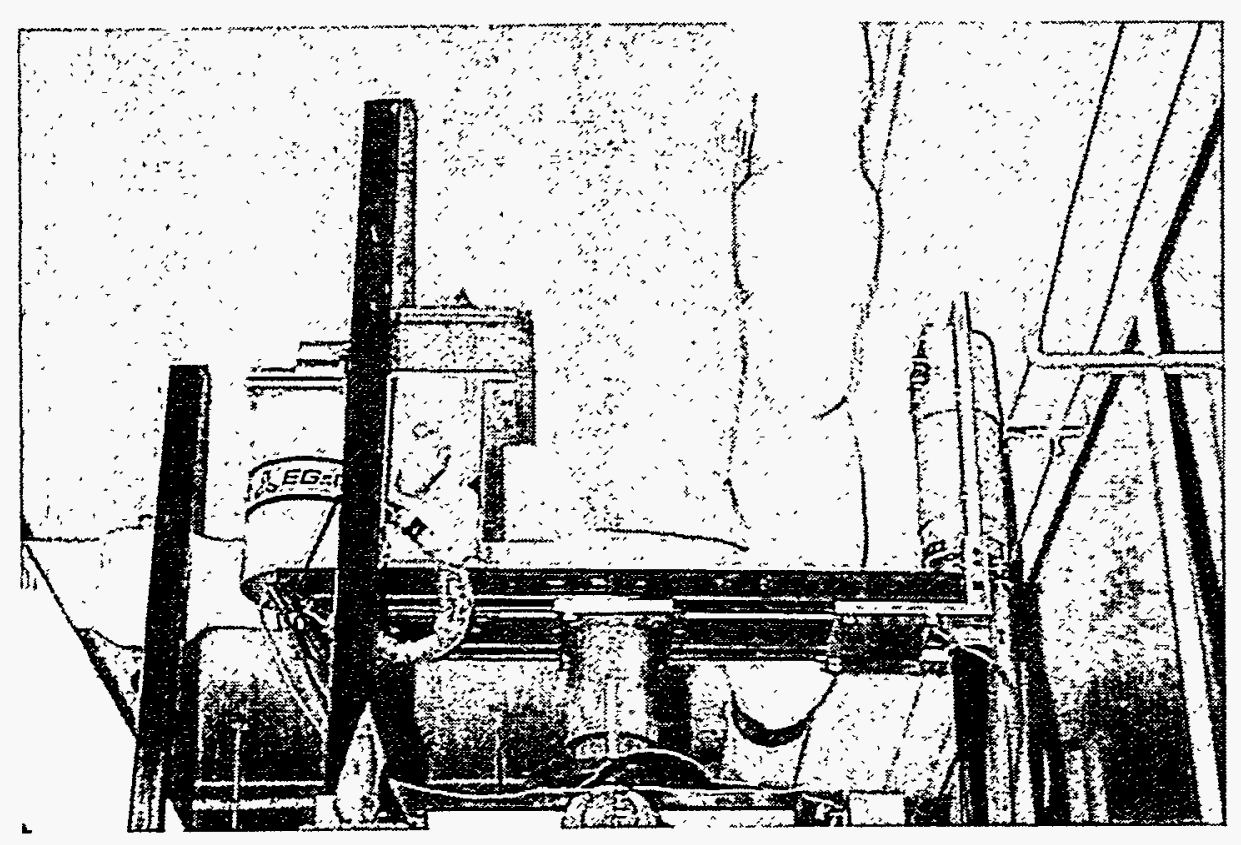

Figure 2. K-edge setup for inspecting a drain trap. The $\mathrm{X}$-ray source is to the right, and the detector is to the left. 


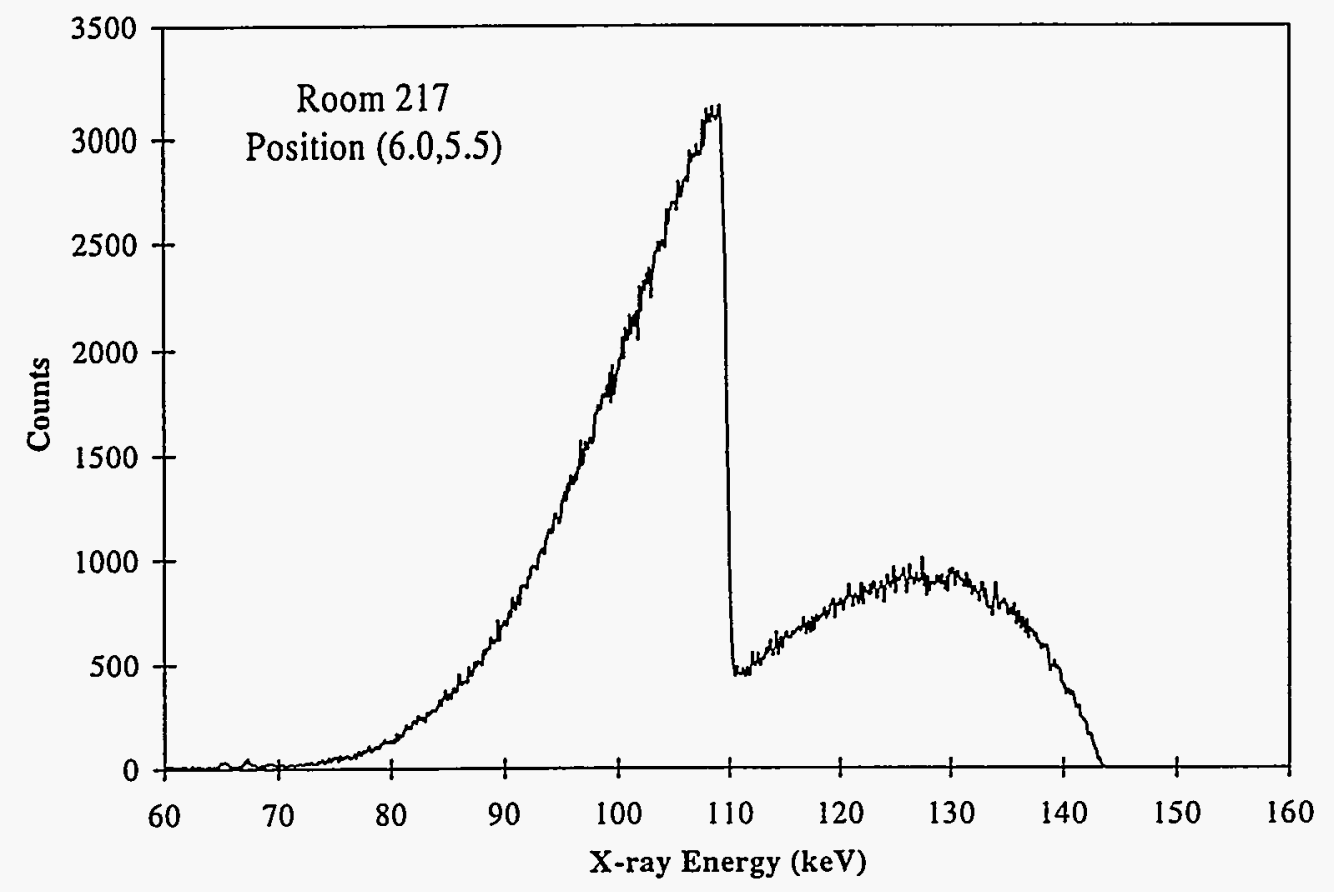

Figure 3. Spectrum showing the highest concentration of thorium found $\left(\sim 0.5 \mathrm{~g} / \mathrm{cm}^{2}\right)$ in the drain trap in room 217.

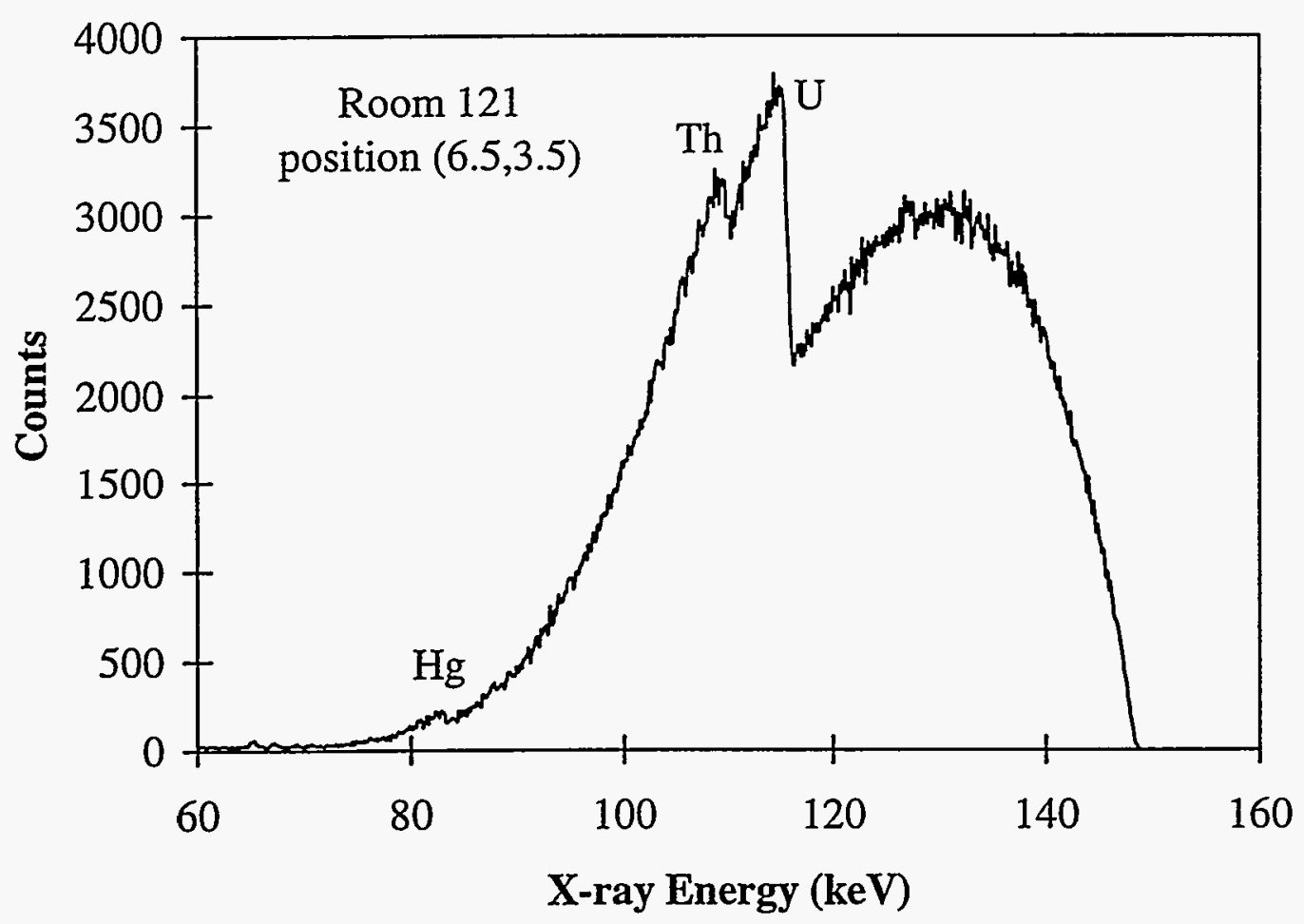

Figure 4. Spectrum from a region of the drain trap in room 121 showing signals for uranium, thorium and mercury. 


\section{RESULTS}

A summary of the findings for each room follows. An image of a pipe with reference grid attached (two inch grid spacing) is followed by tables showing measured contamination concentrations at indicated positions for mercury $(\mathrm{Hg})$, thorium $(\mathrm{Th})$, and uranium (U). Note that the tables do not always have uniform spacing between entries. Uncertainties indicated in the results were calculated based on the statistics of the measurements and represent one standard deviation. In cases where no significant signal was observed, an upper limit at $99.7 \%$ Confidence Level is quoted.

\section{$\underline{\text { Room } 103}$}

To get at the drain lines in this room it was necessary to remove a section of the suspended ceiling. There is a drain trap near the West wall that could be easily accessed with the K-edge system. This pipe travels to the East where it connects with two other drain traps. Because this room had been partitioned off, it was possible to measure only a short distance along this pipe.

Radiographs showed significant scale deposits in the bottom half of the drain trap with several dense wire-like objects standing out. Measurements with a hand-held survey meter showed $5 \mu \mathrm{R} / \mathrm{hr}$ above background at the bottom of the drain trap, $40 \mu \mathrm{R} / \mathrm{hr}$ in a diagonal pipe between the drain trap and the East-West pipe, $80 \mu \mathrm{R} / \mathrm{hr}$ at the transition to the E-W pipe, and $170 \mu \mathrm{R} / \mathrm{hr}$ on the E-W pipe one foot from the transition.

As indicated in the tables below, $\mathrm{K}$-edge measurements indicate no significant contamination in the drain trap. A measurement at the beginning of the diagonal pipe showed $<11.6 \mathrm{mg} / \mathrm{cm}^{2} \mathrm{Hg},<5.3 \mathrm{mg} / \mathrm{cm}^{2} \mathrm{Th}$, and $<9.9 \mathrm{mg} / \mathrm{cm}^{2} \mathrm{U}$. Measurements at the downstream end of the diagonal pipe yielded $<14.8 \mathrm{mg} / \mathrm{cm}^{2} \mathrm{Hg}, 43.9+/-1.7 \mathrm{mg} / \mathrm{cm}^{2} \mathrm{Th}$, and $<7.3 \mathrm{mg} / \mathrm{cm}^{2} \mathrm{U}$. As can be seen from the tables for the E-W pipe there is significant thorium contamination in this region. There is also one location with a small amount of uranium.

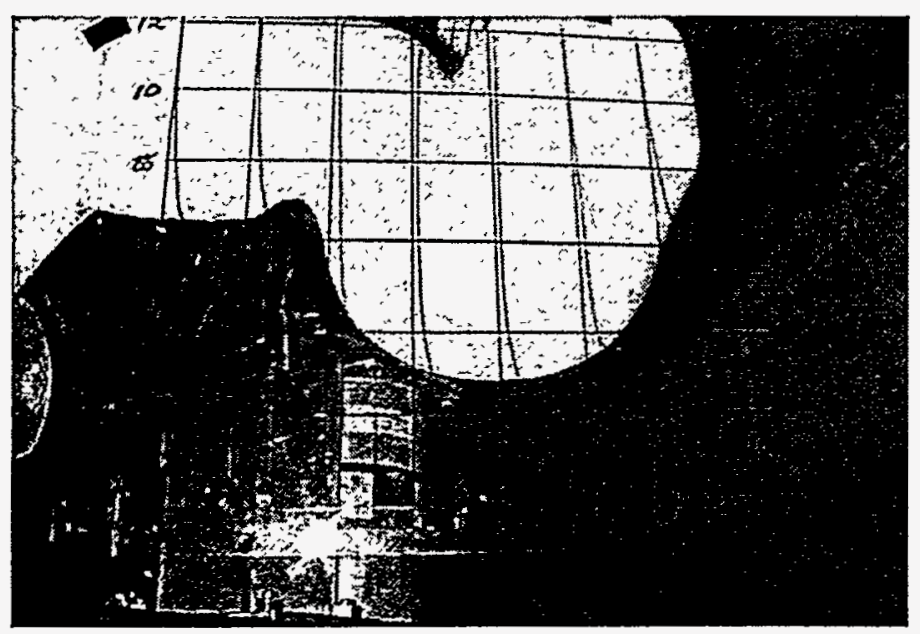

Drain Trap, Room 103 
Table $103 \mathrm{a} . \mathrm{Hg}$. Mercury concentration $\left(\mathrm{mg} / \mathrm{cm}^{2}\right)$ as a function of position.

\begin{tabular}{l|l|l|l|l|l|l|l|l|l}
\hline $\begin{array}{l}\text { Position } \\
\text { (inches) }\end{array}$ & $\mathbf{3 . 0}$ & $\mathbf{4 . 0}$ & $\mathbf{5 . 0}$ & $\mathbf{6 . 0}$ & $\mathbf{7 . 0}$ & $\mathbf{8 . 0}$ & $\mathbf{9 . 0}$ & $\mathbf{1 0 . 0}$ & $\mathbf{1 1 . 0}$ \\
\hline $\mathbf{1 0 . 0}$ & $<78$ & $<4.9$ & & & & & & & \\
\hline $\mathbf{9 . 0}$ & & & & & & & & & \\
\hline $\mathbf{8 . 0}$ & & & & & & & & & \\
\hline $\mathbf{7 . 0}$ & & & & & & & & & \\
\hline $\mathbf{6 . 0}$ & & & $<35.3$ & $<10.4$ & $<9.7$ & $<11.4$ & $<11.6$ & $<17.4$ & $<5.5$ \\
\hline $\mathbf{5 . 5}$ & & & & $<21.3$ & $<6.8$ & $<6.4$ & $<18.9$ & & \\
\hline $\mathbf{5 . 0}$ & & & & $<41.3$ & $<25.0$ & $<16.3$ & & &
\end{tabular}

Table 103 a.Th. Thorium concentration $\left(\mathrm{mg} / \mathrm{cm}^{2}\right)$ as a function of position.

\begin{tabular}{l|l|l|l|l|l|l|l|l|l|l}
\hline $\begin{array}{l}\text { Position } \\
\text { (inches) }\end{array}$ & $\mathbf{3 . 0}$ & $\mathbf{4 . 0}$ & $\mathbf{5 . 0}$ & $\mathbf{6 . 0}$ & $\mathbf{7 . 0}$ & $\mathbf{8 . 0}$ & $\mathbf{9 . 0}$ & $\mathbf{1 0 . 0}$ & $\mathbf{1 1 . 0}$ \\
\hline $\mathbf{1 0 . 0}$ & $<1.0$ & $<1.2$ & & & & & & & \\
\hline $\mathbf{9 . 0}$ & & & & & & & & & \\
\hline $\mathbf{8 . 0}$ & & & & & & & & & \\
\hline $\mathbf{7 . 0}$ & & & & & & & & & \\
\hline $\mathbf{6 . 0}$ & & & $<11.3$ & $<6.2$ & $<6.3$ & $<6.4$ & $<6.7$ & $<4.1$ & $<0.7$ \\
\hline $\mathbf{5 . 5}$ & & & & $<9.5$ & $<6.2$ & $<1.6$ & $<2.8$ & & \\
\hline $\mathbf{5 . 0}$ & & & & $<10.3$ & $<3.3$ & $<7.2$ & & &
\end{tabular}

Table 103a.U. Uranium concentration $\left(\mathrm{mg} / \mathrm{cm}^{2}\right)$ as a function of position.

\begin{tabular}{l|l|l|l|l|l|l|l|l|l}
\hline $\begin{array}{l}\text { Position } \\
\text { (inches) }\end{array}$ & $\mathbf{3 . 0}$ & $\mathbf{4 . 0}$ & $\mathbf{5 . 0}$ & $\mathbf{6 . 0}$ & $\mathbf{7 . 0}$ & $\mathbf{8 . 0}$ & $\mathbf{9 . 0}$ & $\mathbf{1 0 . 0}$ & $\mathbf{1 1 . 0}$ \\
\hline $\mathbf{1 0 . 0}$ & $<36$ & $<3.3$ & & & & & & & \\
\hline $\mathbf{9 . 0}$ & & & & & & & & & \\
\hline $\mathbf{8 . 0}$ & & & & & & & & & \\
\hline $\mathbf{7 . 0}$ & & & & & & & & & \\
\hline $\mathbf{6 . 0}$ & & & $<19.0$ & $<3.4$ & $<9.0$ & $<5.9$ & $<7.4$ & $<9.8$ & $<2.1$ \\
\hline $\mathbf{5 . 5}$ & & & & $<11.5$ & $<8.2$ & $<3.3$ & $<5.4$ & & \\
\hline $\mathbf{5 . 0}$ & & & & $<18.9$ & $<9.2$ & $<12.2$ & & &
\end{tabular}




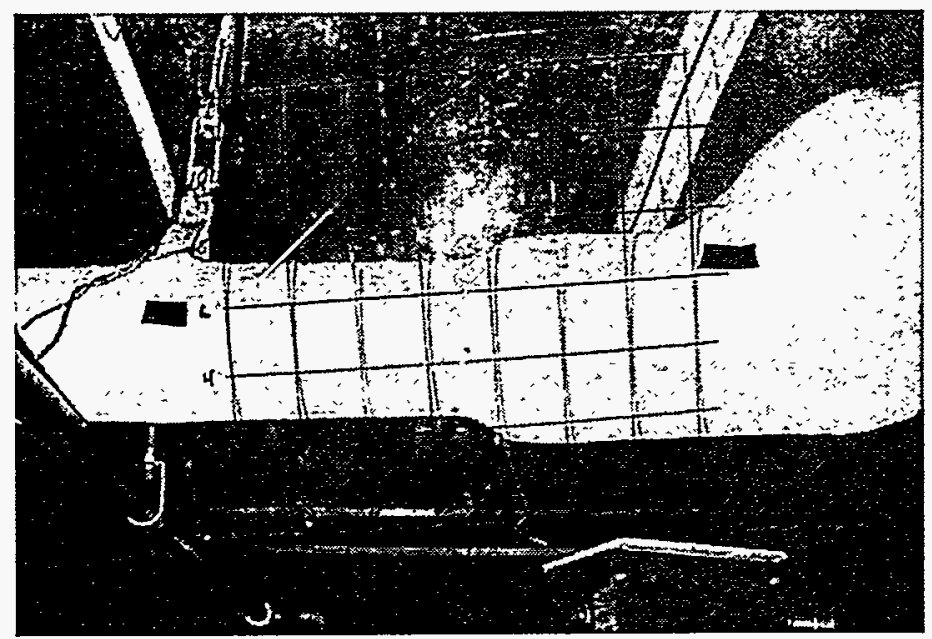

East-West Pipe, Room 103

Table 103b.Hg. Mercury concentration (mg/ $\mathrm{cm}^{2}$ ) as a function of position.

\begin{tabular}{l|l|l|l|l|l|l|l|l}
$\begin{array}{l}\text { Position } \\
\text { (inches) }\end{array}$ & $\mathbf{5 . 0}$ & $\mathbf{6 . 0}$ & $\mathbf{7 . 0}$ & $\mathbf{8 . 0}$ & $\mathbf{9 . 0}$ & $\mathbf{1 0 . 0}$ & $\mathbf{1 2 . 0}$ & $\mathbf{1 4 . 0}$ \\
\hline $\mathbf{6 . 0}$ & & & & $<6.6$ & & $<25.2$ & $<90.6$ & \\
\hline $\mathbf{5 . 5}$ & & $<15.7$ & & $<34.3$ & & $<47.3$ & & \\
\hline $\mathbf{5 . 0}$ & & $<41.3$ & & $<32.2$ & & $<35.1$ & & $<29.6$ \\
\hline $\mathbf{4 . 5}$ & $<58.4$ & $<48.0$ & $<56.4$ & $<47.4$ & $<31.1$ & $<23.1$ & & $<11.2$
\end{tabular}

Table 103b.Th. Thorium concentration $\left(\mathrm{mg} / \mathrm{cm}^{2}\right)$ as a function of position.

\begin{tabular}{l|l|l|l|l|l|l|l|l}
\hline $\begin{array}{l}\text { Position } \\
\text { (inches) }\end{array}$ & $\mathbf{5 . 0}$ & $\mathbf{6 . 0}$ & $\mathbf{7 . 0}$ & $\mathbf{8 . 0}$ & $\mathbf{9 . 0}$ & $\mathbf{1 0 . 0}$ & $\mathbf{1 2 . 0}$ & $\mathbf{1 4 . 0}$ \\
\hline $\mathbf{6 . 0}$ & & & & $\begin{array}{l}4.4 \\
+/-1.2\end{array}$ & & $\begin{array}{l}71.2 \\
+/-3.0\end{array}$ & $<19.7$ & \\
\hline $\mathbf{5 . 5}$ & & $\begin{array}{l}129.4 \\
+/-2.3\end{array}$ & & $\begin{array}{l}200.0 \\
+/-3.8\end{array}$ & & $\begin{array}{l}233.5 \\
+/-5.8\end{array}$ & $\begin{array}{l}236.1 \\
+/-17\end{array}$ & \\
\hline $\mathbf{5 . 0}$ & & 245.9 & & $\begin{array}{l}154.5 \\
+/-3.9\end{array}$ & & $\begin{array}{l}191.9 \\
+/-6.1\end{array}$ & $\begin{array}{l}96.0 \\
+/-19\end{array}$ & $\begin{array}{l}75.3 \\
+/-2.9\end{array}$ \\
\hline $\mathbf{4 . 5}$ & 105.9 & $\begin{array}{l}59.2 \\
+/-5.7\end{array}$ & $\begin{array}{l}67.4 \\
+/-3.0\end{array}$ & $<28.7$ & $<20.5$ & $<17.7$ & & $<1.2$ \\
& $+/-4.2$ & & & & &
\end{tabular}

Table 103b.U. Uranium concentration $\left(\mathrm{mg} / \mathrm{cm}^{2}\right)$ as a function of position.

\begin{tabular}{l|l|l|l|l|l|l|l|l}
\hline $\begin{array}{l}\text { Position } \\
\text { (inches) }\end{array}$ & $\mathbf{5 . 0}$ & $\mathbf{6 . 0}$ & $\mathbf{7 . 0}$ & $\mathbf{8 . 0}$ & $\mathbf{9 . 0}$ & $\mathbf{1 0 . 0}$ & $\mathbf{1 2 . 0}$ & $\mathbf{1 4 . 0}$ \\
\hline $\mathbf{6 . 0}$ & & & & $<3.8$ & & $<19.8$ & $<27.1$ & \\
\hline $\mathbf{5 . 5}$ & & $<13.2$ & & $<18.4$ & & $<25.1$ & & \\
\hline $\mathbf{5 . 0}$ & & $<39.0$ & & $\begin{array}{l}15.5 \\
+1-3.9\end{array}$ & & $<40.0$ & $<50.7$ & $<13.3$ \\
\hline $\mathbf{4 . 5}$ & $<22.9$ & $<19.0$ & $<20.9$ & $<24.0$ & $<36.1$ & $<35.0$ & & $<7.6$
\end{tabular}




\section{Room 121}

There is a drain trap in the center of the room and one near the East wall that both tie into a pipe going into the South wall. It was possible to access only the drain trap at the center of the room. A radiograph showed significant variation in density throughout the drain trap. A measurement with a hand-held survey meter showed $10 \mu \mathrm{R} / \mathrm{hr}$ above background on the downstream side of the trap.

$\mathrm{K}$-edge measurements indicate significant deposits of mercury, thorium and uranium in this trap. Mercury and uranium are found throughout the trap, whereas thorium appears to be concentrated in a small region. Several measurements were also made on the straight section of pipe just downstream from this trap. No significant contamination was found in this region, with limits of $<11 \mathrm{mg} / \mathrm{cm}^{2} \mathrm{Hg},<4 \mathrm{mg} / \mathrm{cm}^{2} \mathrm{Th}$, and $<7 \mathrm{mg} / \mathrm{cm}^{2} \mathrm{U}$.

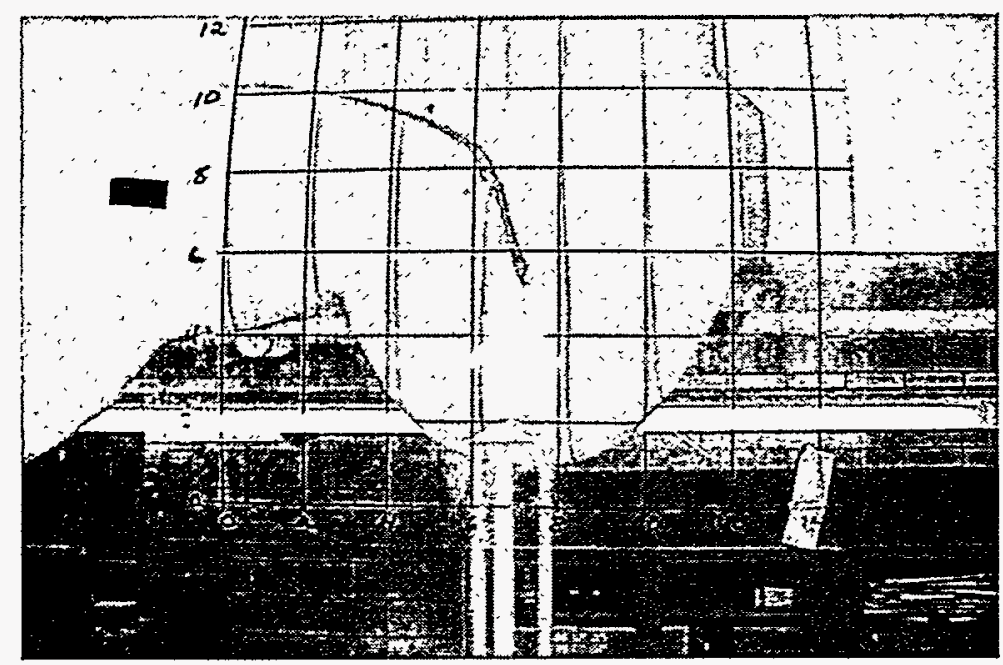

Center Drain Trap, Room 121

Table 121.Hg. Mercury concentration $\left(\mathrm{mg} / \mathrm{cm}^{2}\right)$ as a function of position.

\begin{tabular}{|c|c|c|c|c|c|c|c|c|c|c|}
\hline $\begin{array}{l}\text { Position } \\
\text { (inches) }\end{array}$ & 4.5 & 5.0 & 5.5 & 6.0 & 6.5 & 7.0 & 7.5 & 8.0 & 9.0 & 10.0 \\
\hline 4.0 & $\begin{array}{l}31.1 \\
+/-7.2\end{array}$ & $\begin{array}{l}55.2 \\
+1-6.1\end{array}$ & & $\begin{array}{l}62.2 \\
+/-12\end{array}$ & & $\begin{array}{l}28.7 \\
+1-6.1\end{array}$ & & $<18.5$ & $\begin{array}{l}59.1 \\
+/-7.9\end{array}$ & $<44.4$ \\
\hline 3.5 & & $<61.7$ & $\begin{array}{l}32.0 \\
+/-6.7\end{array}$ & $\begin{array}{l}75.8 \\
+/-7.0 \\
\end{array}$ & $\begin{array}{l}82.3 \\
+/-8.9 \\
\end{array}$ & $\begin{array}{l}78.9 \\
+/-9.9 \\
\end{array}$ & & $\begin{array}{l}37.7 \\
+/-7.0 \\
\end{array}$ & $<127$ & $<59.5$ \\
\hline 3.0 & & $<69$ & & $\begin{array}{l}51.0 \\
+/-11 \\
\end{array}$ & & $\begin{array}{l}39.6 \\
+/-15\end{array}$ & & $\begin{array}{l}69.2 \\
+/-8.8\end{array}$ & & \\
\hline 2.5 & & & & & & $<90$ & $\begin{array}{l}72.0 \\
+/-13\end{array}$ & & & \\
\hline
\end{tabular}


Table 121.Th. Thorium concentration $\left(\mathrm{mg} / \mathrm{cm}^{2}\right)$ as a function of position.

\begin{tabular}{l|l|l|l|l|l|l|l|l|l|l}
$\begin{array}{l}\text { Position } \\
\text { (inches) }\end{array}$ & 4.5 & $\mathbf{5 . 0}$ & $\mathbf{5 . 5}$ & $\mathbf{6 . 0}$ & $\mathbf{6 . 5}$ & $\mathbf{7 . 0}$ & $\mathbf{7 . 5}$ & $\mathbf{8 . 0}$ & $\mathbf{9 . 0}$ & $\mathbf{1 0 . 0}$ \\
\hline $\mathbf{4 . 0}$ & $<4.8$ & $<6.2$ & & $<20.6$ & & $<8.5$ & & $<5.3$ & $<20.0$ & $<8.6$ \\
\hline 3.5 & & $<9.8$ & $<3.4$ & $<5.6$ & $\begin{array}{l}34.8 \\
+/-3.2\end{array}$ & $\begin{array}{l}18.9 \\
+/-3.3\end{array}$ & $<80$ & $<10.3$ & $<18.9$ & $<8.7$ \\
\hline $\mathbf{3 . 0}$ & & $<24.2$ & & $<10.1$ & & $\begin{array}{l}45.5 \\
+/-4.6\end{array}$ & & $<14.6$ & & \\
\hline $\mathbf{2 . 5}$ & & & & & & $<32.8$ & $<7.1$ & & &
\end{tabular}

Table 121.U. Uranium concentration $\left(\mathrm{mg} / \mathrm{cm}^{2}\right)$ as a function of position.

\begin{tabular}{l|l|l|l|l|l|l|l|l|l|l}
\hline $\begin{array}{l}\text { Position } \\
\text { (inches) }\end{array}$ & 4.5 & $\mathbf{5 . 0}$ & $\mathbf{5 . 5}$ & $\mathbf{6 . 0}$ & $\mathbf{6 . 5}$ & $\mathbf{7 . 0}$ & $\mathbf{7 . 5}$ & $\mathbf{8 . 0}$ & $\mathbf{9 . 0}$ & $\mathbf{1 0 . 0}$ \\
\hline $\mathbf{4 . 0}$ & $<16.5$ & $\begin{array}{l}14.4 \\
+/-2.5\end{array}$ & & $\begin{array}{l}16.9 \\
+/-5.4\end{array}$ & & $\begin{array}{l}7.7 \\
+/-2.9\end{array}$ & & $<11.2$ & $<23.8$ & $<14.7$ \\
\hline $\mathbf{3 . 5}$ & & $<19.6$ & $<14.6$ & $\begin{array}{l}12.8 \\
+/-2.5\end{array}$ & $\begin{array}{l}178.9 \\
+/-3.3\end{array}$ & $\begin{array}{l}122.5 \\
+/-3.4\end{array}$ & $\begin{array}{l}107 \\
+/-20\end{array}$ & $<15.6$ & $<28.3$ & $<15.1$ \\
\hline $\mathbf{3 . 0}$ & & 20.8 & & $\begin{array}{l}20.3 \\
+/-3.5\end{array}$ & & $\begin{array}{l}239.4 \\
+/-4.9\end{array}$ & & $\begin{array}{l}26.2 \\
+/-3.1\end{array}$ & & \\
\hline $\mathbf{2 . 5}$ & & & & & & $<50$ & $<19.5$ & & &
\end{tabular}

\section{$\underline{\text { Room } 129}$}

There is an easily accessible drain trap in the Southwest corner of the room. A radiograph shows scale buildup towards the downstream side of the trap with density variations throughout the region. Hand-held survey meter readings indicate 5-10 $\mu \mathrm{R} / \mathrm{hr}$ above background in the trap and in the straight section of pipe downstream.

K-edge measurements indicate significant deposits of mercury in the trap, but show no evidence for thorium or uranium. There was also a strong signal for lead, which, along with the mercury, could explain the observed strong absorption of X-rays. This resulted in greater uncertainty in the thorium and uranium measurements. The observed limits for these elements are consistent with the 5-10 $\mu \mathrm{R} / \mathrm{hr}$ survey meter readings. A measurement on the straight section of pipe near the joint indicated $<20 \mathrm{mg} / \mathrm{cm}^{2} \mathrm{Hg},<4 \mathrm{mg} / \mathrm{cm}^{2} \mathrm{Th}$, and $<10 \mathrm{mg} / \mathrm{cm}^{2} \mathrm{U}$. 


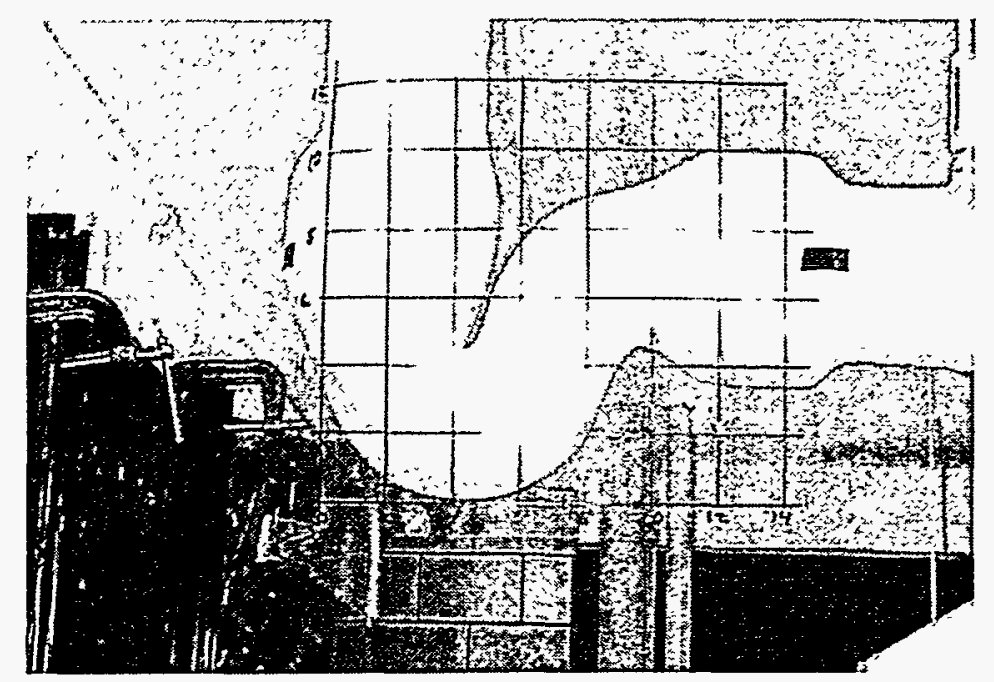

Drain Trap, Room 129

Table 129. Hg. Mercury concentration $\left(\mathrm{mg} / \mathrm{cm}^{2}\right.$ ) as a function of position.

\begin{tabular}{l|l|l|l|l|l}
\hline $\begin{array}{l}\text { Position } \\
\text { (inches) }\end{array}$ & $\mathbf{2 . 0}$ & $\mathbf{3 . 0}$ & $\mathbf{4 . 0}$ & $\mathbf{5 . 0}$ & $\mathbf{6 . 0}$ \\
\hline $\mathbf{4 . 0}$ & & & $<14.6$ & $176+/-5$ & $121+/-27$ \\
\hline $\mathbf{3 . 0}$ & $<163$ & $<127$ & $24.4+/-2.4$ & $<136$ & $<198$
\end{tabular}

Table 129.Th. Thorium concentration $\left(\mathrm{mg} / \mathrm{cm}^{2}\right)$ as a function of position.

\begin{tabular}{l|l|l|l|l|l}
\hline $\begin{array}{l}\text { Position } \\
\text { (inches) }\end{array}$ & $\mathbf{2 . 0}$ & $\mathbf{3 . 0}$ & $\mathbf{4 . 0}$ & $\mathbf{5 . 0}$ & $\mathbf{6 . 0}$ \\
\hline $\mathbf{4 . 0}$ & & & $<4.4$ & $<3.9$ & \\
\hline $\mathbf{3 . 0}$ & $<35.1$ & $<12.8$ & $<6.5$ & $<97$ & $<34.5$
\end{tabular}

Table 129.U. Uranium concentration $\left(\mathrm{mg} / \mathrm{cm}^{2}\right)$ as a function of position.

\begin{tabular}{l|l|l|l|l|l}
$\begin{array}{l}\text { Position } \\
\text { (inches) }\end{array}$ & $\mathbf{2 . 0}$ & $\mathbf{3 . 0}$ & 4.0 & $\mathbf{5 . 0}$ & $\mathbf{6 . 0}$ \\
\hline $\mathbf{4 . 0}$ & & & $<4.6$ & $<13.5$ & \\
\hline $\mathbf{3 . 0}$ & $<39.6$ & $<42.5$ & $<6.2$ & $<81.6$ & $<67.7$
\end{tabular}

\section{$\underline{\text { Room } 203}$}

The drain trap is situated next to a support beam, which makes access very difficult. Thus, it was also hard to obtain a good radiograph of the trap. Significant density variations were observed in the small region that could be adequately radiographed. Hand-held survey meter readings indicated $10-15 \mu \mathrm{R} / \mathrm{hr}$ above background in the trap. K-edge measurements indicate $10-70 \mathrm{mg} / \mathrm{cm}^{2}$ thorium at the bottom of the drain trap. 


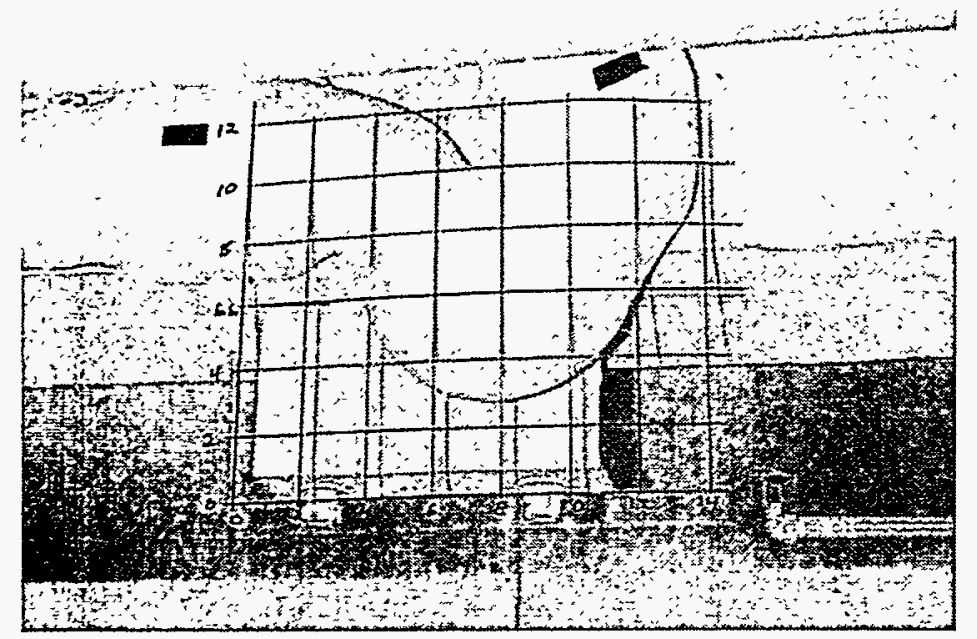

Drain Trap, Room 203

Table 203.Hg. Mercury concentration $\left(\mathrm{mg} / \mathrm{cm}^{2}\right)$ as a function of position.

\begin{tabular}{l|l|l|l|l|l}
\hline $\begin{array}{l}\text { Position } \\
\text { (inches) }\end{array}$ & $\mathbf{5 . 0}$ & $\mathbf{6 . 0}$ & $\mathbf{7 . 0}$ & $\mathbf{8 . 0}$ & $\mathbf{8 . 5}$ \\
\hline $\mathbf{7 . 0}$ & & $<14.2$ & $<14.7$ & $<25.8$ & \\
\hline $\mathbf{6 . 5}$ & $<17.5$ & $<25.1$ & $<22.0$ & & $<28.8$ \\
\hline $\mathbf{5 . 0}$ & & $<27.1$ & & $<84.1$ &
\end{tabular}

Table 203.Th. Thorium concentration $\left(\mathrm{mg} / \mathrm{cm}^{2}\right)$ as a function of position.

\begin{tabular}{l|l|l|l|l|l}
\hline $\begin{array}{l}\text { Position } \\
\text { (inches) }\end{array}$ & $\mathbf{5 . 0}$ & $\mathbf{6 . 0}$ & $\mathbf{7 . 0}$ & $\mathbf{8 . 0}$ & $\mathbf{8 . 5}$ \\
\hline $\mathbf{7 . 0}$ & & $9.7+/-2.0$ & $6.8+/-1.6$ & $8.9+/-1.9$ & \\
\hline $\mathbf{6 . 5}$ & $<10.1$ & $55.5+/-2.9$ & $60.9+/-3.2$ & & $12.0+/-2.1$ \\
\hline $\mathbf{5 . 0}$ & & $<5.1$ & & $<12.4$ &
\end{tabular}

Table 203.U. Uranium concentration $\left(\mathrm{mg} / \mathrm{cm}^{2}\right)$ as a function of position.

\begin{tabular}{l|l|l|l|l|l}
$\begin{array}{l}\text { Position } \\
\text { (inches) }\end{array}$ & $\mathbf{5 . 0}$ & $\mathbf{6 . 0}$ & $\mathbf{7 . 0}$ & $\mathbf{8 . 0}$ & $\mathbf{8 . 5}$ \\
\hline $\mathbf{7 . 0}$ & & $<11.5$ & $<8.3$ & $<12.2$ & \\
\hline $\mathbf{6 . 5}$ & $<8.2$ & $<12.6$ & $<13.5$ & & $<10.0$ \\
\hline $\mathbf{5 . 0}$ & & $<12.6$ & & $<19.9$ &
\end{tabular}

\section{$\underline{\text { Room } 209}$}

The drain trap is near a support beam that limits access. The drain line extends to the South side of the room where it connects into another pipe. A radiograph showed some scale buildup but no strong features. Hand-held survey meter measurements showed nothing above background. K-edge measurements are consistent with this, showing no 
significant contamination. A measurement on the straight section of the pipe just downstream of the trap indicated $<9 \mathrm{mg} / \mathrm{cm}^{2} \mathrm{Hg},<6 \mathrm{mg} / \mathrm{cm}^{2} \mathrm{Th}$, and $<8 \mathrm{mg} / \mathrm{cm}^{2} \mathrm{U}$.

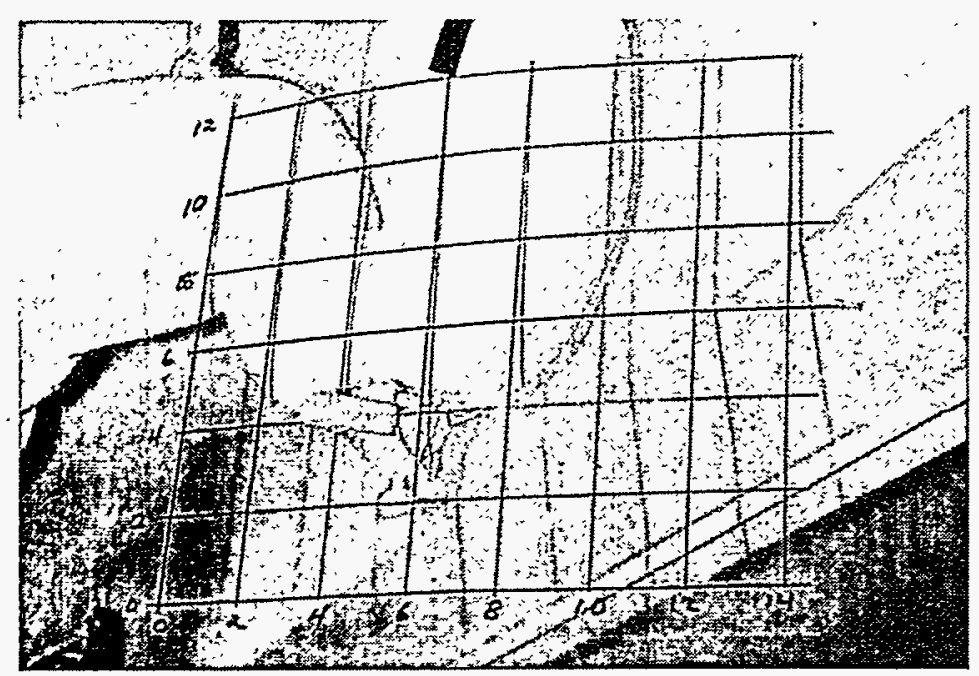

Drain Trap, Room 209

Table 209.Hg. Mercury concentration $\left(\mathrm{mg} / \mathrm{cm}^{2}\right)$ as a function of position.

\begin{tabular}{l|l|l}
\hline $\begin{array}{l}\text { Position } \\
\text { (inches) }\end{array}$ & $\mathbf{3 . 0}$ & $\mathbf{5 . 0}$ \\
\hline $\mathbf{8 . 5}$ & & $<10.0$ \\
\hline $\mathbf{8 . 0}$ & $<35.2$ & $<23.8$ \\
\hline $\mathbf{7 . 2 5}$ & & $<16.6$ \\
\hline $\mathbf{6 . 7 5}$ & $<90$ & $<97.5$ \\
\hline $\mathbf{6 . 0}$ & & $<75$
\end{tabular}

Table 209.Th. Thorium concentration $\left(\mathrm{mg} / \mathrm{cm}^{2}\right)$ as a function of position.

\begin{tabular}{l|l|l}
$\begin{array}{l}\text { Position } \\
\text { (inches) }\end{array}$ & $\mathbf{3 . 0}$ & $\mathbf{5 . 0}$ \\
\hline $\mathbf{8 . 5}$ & & $<1.5$ \\
\hline $\mathbf{8 . 0}$ & $<5.7$ & $<6.2$ \\
\hline $\mathbf{7 . 2 5}$ & & $<6.0$ \\
\hline $\mathbf{6 . 7 5}$ & $<69$ & $<4.1$ \\
\hline $\mathbf{6 . 0}$ & & $<10.1$
\end{tabular}

Table 209.U. Uranium concentration $\left(\mathrm{mg} / \mathrm{cm}^{2}\right)$ as a function of position.

\begin{tabular}{l|l|l}
\hline $\begin{array}{l}\text { Position } \\
\text { (inches) }\end{array}$ & $\mathbf{3 . 0}$ & $\mathbf{5 . 0}$ \\
\hline $\mathbf{8 . 5}$ & & $<7.1$ \\
\hline $\mathbf{8 . 0}$ & $<16.3$ & $<17.0$ \\
\hline $\mathbf{7 . 2 5}$ & & $<10.0$ \\
\hline $\mathbf{6 . 7 5}$ & $<87.3$ & $<20.0$ \\
\hline $\mathbf{6 . 0}$ & & $<22.0$
\end{tabular}




\section{$\underline{\text { Room } 217}$}

The drain trap is in the Southwest corner of the room. The line goes through several bends and joints before entering a straight section. It then travels East to the center of the room, and then turns South and intersects with a vertical pipe. A radiograph shows significant scale buildup, especially on the downstream side of the trap. Hand-held survey meter readings gave high values of $300 \mu \mathrm{R} / \mathrm{hr}$ at the drain trap, $70 \mu \mathrm{R} / \mathrm{hr}$ around the bends downstream of the trap, and $300-400 \mu \mathrm{R} / \mathrm{hr}$ at the straight section.

K-edge measurements indicate substantial thorium deposits in this drain trap (as high as $0.5 \mathrm{~g} / \mathrm{cm}^{2}$ ), and moderate thorium contamination in the joint region downstream of the trap. The joints also contained substantial amounts of lead (likely from soldering) which made it quite difficult to obtain adequate statistics in these regions. It was not possible to position the K-edge system on the straight section of the pipe.

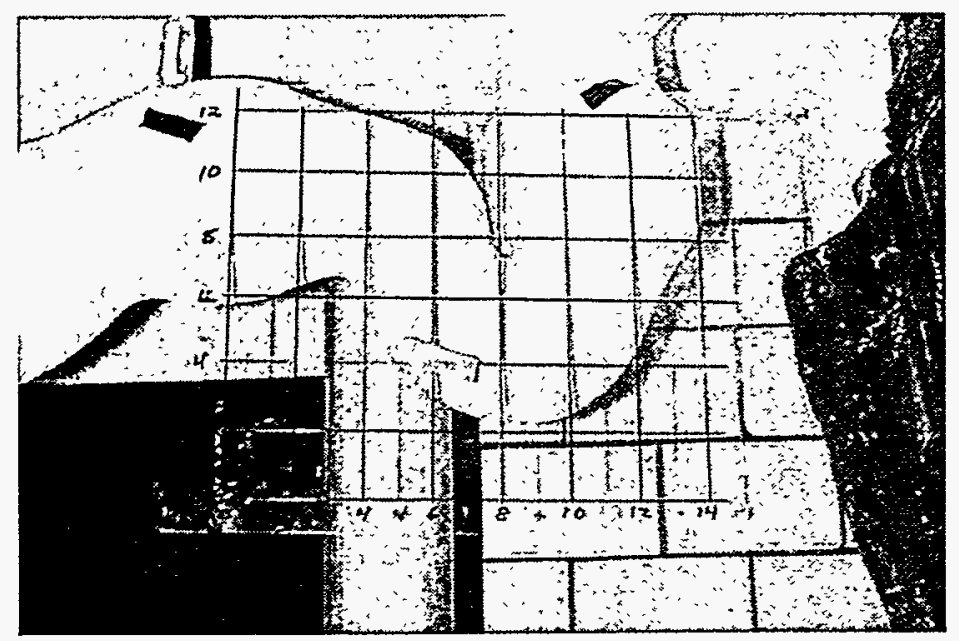

Drain Trap, Room 217

Table $217 \mathrm{a} . \mathrm{Hg}$. Mercury concentration $\left(\mathrm{mg} / \mathrm{cm}^{2}\right)$ as a function of position.

\begin{tabular}{l|l|l|l|l|l|l|l|l|l|l|l|l}
\hline $\begin{array}{l}\text { Position } \\
\text { (inches) }\end{array}$ & $\mathbf{5 . 0}$ & $\mathbf{5 . 5}$ & $\mathbf{6 . 0}$ & $\mathbf{6 . 5}$ & $\mathbf{7 . 0}$ & $\mathbf{7 . 5}$ & $\mathbf{8 . 0}$ & $\mathbf{8 . 5}$ & $\mathbf{9 . 0}$ & $\mathbf{9 . 5}$ & $\mathbf{1 0 . 0}$ & $\mathbf{1 0 . 5}$ \\
\hline $\mathbf{6 . 5}$ & & $<13.0$ & & $<9.5$ & & $<8.7$ & & $<15.6$ & & $<11.7$ & & $<6.6$ \\
\hline $\mathbf{5 . 5}$ & $<24.3$ & $<38.7$ & $<32.9$ & $<37.3$ & $<17.4$ & $<4.6$ & $<3.2$ & $<6.3$ & $<7.9$ & $<6.6$ & $<11.3$ & $<10.2$ \\
\hline $\mathbf{4 . 5}$ & & & & $<5.6$ & $<49.7$ & & $<34.5$ & $<35.9$ & & $<41.8$ & &
\end{tabular}


Table 217a.Th. Thorium concentration $\left(\mathrm{mg} / \mathrm{cm}^{2}\right)$ as a function of position.

\begin{tabular}{|c|c|c|c|c|c|c|c|c|c|c|c|c|}
\hline $\begin{array}{l}\text { Position } \\
\text { (inches) }\end{array}$ & 5.0 & 5.5 & 6.0 & 6.5 & 7.0 & 7.5 & 8.0 & 8.5 & 9.0 & 9.5 & 10.0 & 10.5 \\
\hline 6.5 & & $\begin{array}{l}90.4 \\
+/-2.9 \\
\end{array}$ & & $\begin{array}{l}45.5 \\
+/-1.7 \\
\end{array}$ & & $\begin{array}{l}22.0 \\
+l-1.6 \\
\end{array}$ & & $\begin{array}{l}43.2 \\
+-2.4 \\
\end{array}$ & & $\begin{array}{l}43.1 \\
+-2.0 \\
\end{array}$ & & $\begin{array}{l}3.4 \\
+1-1.2 \\
\end{array}$ \\
\hline 5.5 & $<19.2$ & $\begin{array}{l}205.4 \\
+/-4.6 \\
\end{array}$ & $\begin{array}{l}536 \\
+l-7.3 \\
\end{array}$ & $\begin{array}{l}320 \\
+1-5.0 \\
\end{array}$ & $\begin{array}{r}104.9 \\
+/ 2.6 \\
\end{array}$ & $\begin{array}{l}15.3 \\
+/-1.1 \\
\end{array}$ & $\begin{array}{l}10.8 \\
+/-0.9\end{array}$ & $\begin{array}{l}7.7 \\
+1-0.8 \\
\end{array}$ & $\begin{array}{l}11.1 \\
+/-0.9 \\
\end{array}$ & $\begin{array}{l}13.8 \\
+/-0.9 \\
\end{array}$ & \begin{tabular}{|l|}
23.1 \\
$+/-1.7$ \\
\end{tabular} & $\begin{array}{l}86.1 \\
++-2.8 \\
\end{array}$ \\
\hline 4.5 & & & & $\begin{array}{l}34.1 \\
+/-7.0\end{array}$ & $\begin{array}{l}154.5 \\
+/-7.8\end{array}$ & & $\begin{array}{l}139.8 \\
+/-5.4\end{array}$ & $\begin{array}{l}96.1 \\
+/-4.2\end{array}$ & & $\begin{array}{l}14.7 \\
+/-7.7\end{array}$ & & \\
\hline
\end{tabular}

Table 217a.U. Uranium concentration $\left(\mathrm{mg} / \mathrm{cm}^{2}\right)$ as a function of position.

\begin{tabular}{l|l|l|l|l|l|l|l|l|l|l|l|l}
\hline $\begin{array}{l}\text { Position } \\
\text { (inches) }\end{array}$ & $\mathbf{5 . 0}$ & $\mathbf{5 . 5}$ & $\mathbf{6 . 0}$ & $\mathbf{6 . 5}$ & $\mathbf{7 . 0}$ & $\mathbf{7 . 5}$ & $\mathbf{8 . 0}$ & $\mathbf{8 . 5}$ & $\mathbf{9 . 0}$ & $\mathbf{9 . 5}$ & $\mathbf{1 0 . 0}$ & $\mathbf{1 0 . 5}$ \\
\hline $\mathbf{6 . 5}$ & & $<10.1$ & & $<7.5$ & & $<4.7$ & & $<13.2$ & & $<6.1$ & & $<4.8$ \\
\hline $\mathbf{5 . 5}$ & $<5.0$ & $<30.6$ & $<44.2$ & $<25.3$ & $<14.4$ & $<4.5$ & $<1.5$ & $<1.0$ & $<3.5$ & $<6.1$ & $<5.3$ & $<18.9$ \\
\hline 4.5 & & & & $<25.7$ & $<46.3$ & & $<31.8$ & $<15.4$ & & $<21.3$ & &
\end{tabular}

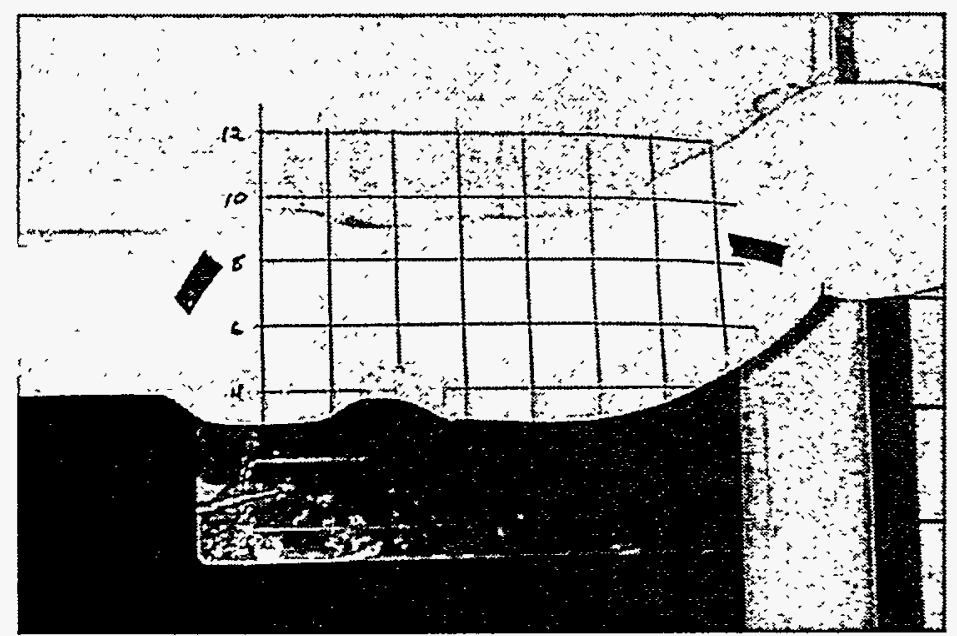

Joint Region, Room 217

Table $217 \mathrm{~b}$. Hg. Mercury concentration $\left(\mathrm{mg} / \mathrm{cm}^{2}\right)$ as a function of position.

\begin{tabular}{l|l|l|l}
\hline $\begin{array}{l}\text { Position } \\
\text { (inches) }\end{array}$ & $\mathbf{8 . 0}$ & $\mathbf{1 0 . 0}$ & $\mathbf{1 2 . 0}$ \\
\hline $\mathbf{8 . 0}$ & $<15.3$ & $<8.0$ & $<41.2$ \\
\hline $\mathbf{6 . 0}$ & $<43.5$ & $<15.3$ &
\end{tabular}

Table 217b.Th. Thorium concentration $\left(\mathrm{mg} / \mathrm{cm}^{2}\right)$ as a function of position.

\begin{tabular}{l|l|l|l}
\hline $\begin{array}{l}\text { Position } \\
\text { (inches) }\end{array}$ & $\mathbf{8 . 0}$ & $\mathbf{1 0 . 0}$ & $\mathbf{1 2 . 0}$ \\
\hline $\mathbf{8 . 0}$ & $<16.4$ & $5.6+/-1.6$ & $64.0+/-4.8$ \\
\hline $\mathbf{6 . 0}$ & $34.5+/-4.6$ & $<6.1$ &
\end{tabular}


Table 217b.U. Uranium concentration $\left(\mathrm{mg} / \mathrm{cm}^{2}\right)$ as a function of position.

\begin{tabular}{l|l|l|l}
\hline $\begin{array}{l}\text { Position } \\
\text { (inches) }\end{array}$ & $\mathbf{8 . 0}$ & $\mathbf{1 0 . 0}$ & $\mathbf{1 2 . 0}$ \\
\hline $\mathbf{8 . 0}$ & $<35.1$ & $<6.5$ & $<18.2$ \\
\hline $\mathbf{6 . 0}$ & $<22.5$ & $<22.1$ &
\end{tabular}

\section{$\underline{\text { Room } 227}$}

This drain trap offers easy access in the center of the room. The line travels to the West wall and then along this wall to the South. A radiograph shows substantial scale buildup at the bottom of the trap and numerous high-density regions including several wire-like objects. Hand-held survey meter readings indicate 5-10 $\mu \mathrm{R} / \mathrm{hr}$ above background at the trap and $10 \mu \mathrm{R} / \mathrm{hr}$ at the joint downstream of the trap.

K-edge measurements indicate a small amount of mercury in the trap, but no other significant contamination. This is at odds with the indications from the hand-held survey meter. It could be that the contamination is localized in small regions, and thus was missed in the rather coarse sampling of the $\mathrm{K}$-edge measurements. (The radiograph indicated significant local density variations.)

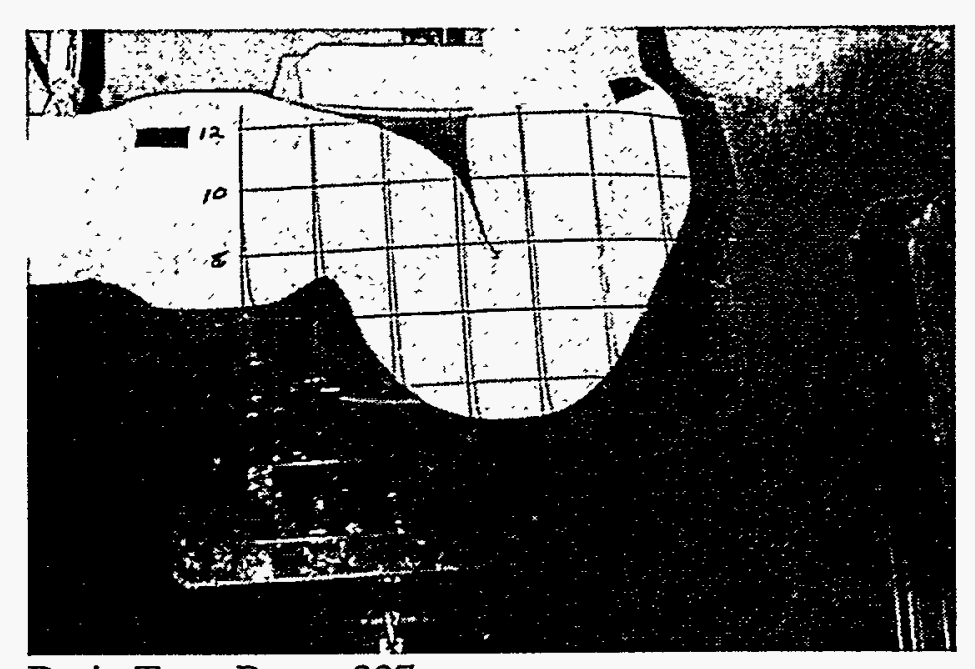

Drain Trap, Room 227

Table 227a.Hg. Mercury concentration $\left(\mathrm{mg} / \mathrm{cm}^{2}\right)$ as a function of position.

\begin{tabular}{l|l|l|l|l|l|l}
\hline $\begin{array}{l}\text { Position } \\
\text { (inches) }\end{array}$ & $\mathbf{5 . 0}$ & $\mathbf{6 . 0}$ & $\mathbf{7 . 0}$ & $\mathbf{8 . 0}$ & $\mathbf{9 . 0}$ & $\mathbf{1 0 . 0}$ \\
\hline $\mathbf{7 . 0}$ & $<13.2$ & $<8.2$ & $<9.2$ & $<10.1$ & $<8.9$ & $<17.8$ \\
\hline $\mathbf{6 . 0}$ & $<21.0$ & $\begin{array}{l}11.5 \\
+1-3.3\end{array}$ & $<6.6$ & & & \\
\hline $\mathbf{5 . 5}$ & & & $<11.1$ & $<21.0$ & & \\
\hline $\mathbf{5 . 0}$ & & & $<45.1$ & $<50.1$ & $<21.4$ &
\end{tabular}


Table 227a.Th. Thorium concentration $\left(\mathrm{mg} / \mathrm{cm}^{2}\right)$ as a function of position.

\begin{tabular}{l|l|l|l|l|l|l}
\hline $\begin{array}{l}\text { Position } \\
\text { (inches) }\end{array}$ & $\mathbf{5 . 0}$ & $\mathbf{6 . 0}$ & $\mathbf{7 . 0}$ & $\mathbf{8 . 0}$ & $\mathbf{9 . 0}$ & $\mathbf{1 0 . 0}$ \\
\hline $\mathbf{7 . 0}$ & $<3.9$ & $<3.5$ & $<7.0$ & $<3.2$ & $<1.8$ & $<5.4$ \\
\hline $\mathbf{6 . 0}$ & $<4.3$ & $<2.7$ & $<1.8$ & & & \\
\hline $\mathbf{5 . 5}$ & & & $<6.1$ & $<7.2$ & & \\
\hline $\mathbf{5 . 0}$ & & & $<1.0$ & $<8.9$ & $<3.9$ &
\end{tabular}

Table 227a.U. Uranium concentration $\left(\mathrm{mg} / \mathrm{cm}^{2}\right)$ as a function of position.

\begin{tabular}{l|l|l|l|l|l|l}
\hline $\begin{array}{l}\text { Position } \\
\text { (inches) }\end{array}$ & $\mathbf{5 . 0}$ & $\mathbf{6 . 0}$ & $\mathbf{7 . 0}$ & $\mathbf{8 . 0}$ & $\mathbf{9 . 0}$ & $\mathbf{1 0 . 0}$ \\
\hline $\mathbf{7 . 0}$ & $<3.0$ & $<2.0$ & $<6.9$ & $<3.9$ & $<5.1$ & $<5.8$ \\
\hline $\mathbf{6 . 0}$ & $<30.7$ & $<4.2$ & $<3.5$ & & & \\
\hline $\mathbf{5 . 5}$ & & & $<15.6$ & $<9.0$ & & \\
\hline $\mathbf{5 . 0}$ & & & $<9.2$ & $<18.5$ & $<15.0$ &
\end{tabular}

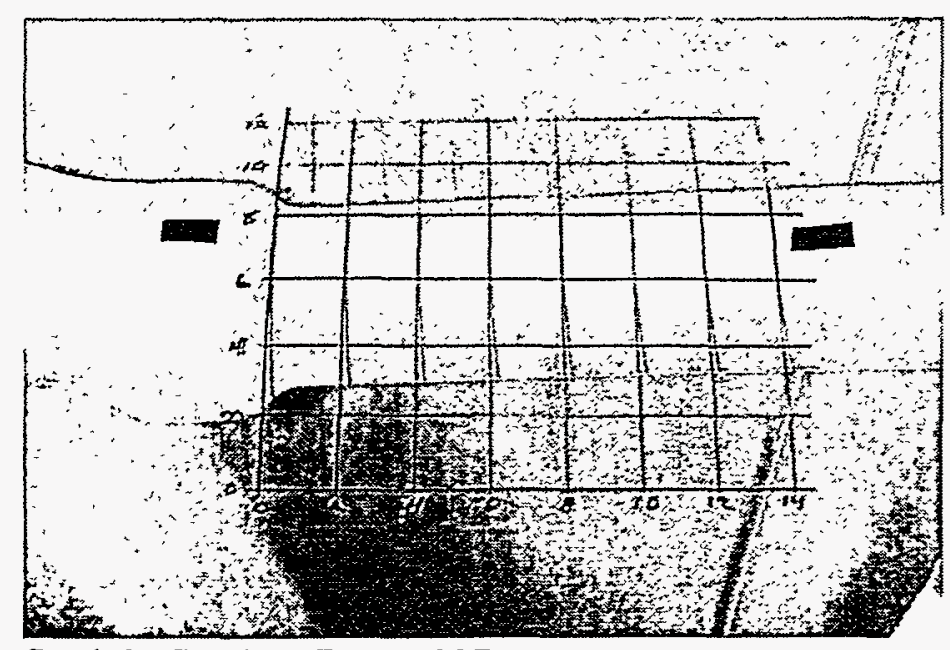

Straight Section, Room 227

Table 227b. Hg. Mercury concentration $\left(\mathrm{mg} / \mathrm{cm}^{2}\right)$ as a function of position.

\begin{tabular}{l|l|l}
$\begin{array}{l}\text { Position } \\
\text { (inches) }\end{array}$ & $\mathbf{0 . 0}$ & $\mathbf{1 . 0}$ \\
\hline $\mathbf{7 . 0}$ & $<7.1$ & $<6.5$ \\
\hline $\mathbf{6 . 5}$ & $<7.0$ & $<9.2$ \\
\hline $\mathbf{6 . 0}$ & $<14.4$ & $<20.7$
\end{tabular}

Table 227b.Th. Thorium concentration $\left(\mathrm{mg} / \mathrm{cm}^{2}\right)$ as a function of position.

\begin{tabular}{l|l|l}
$\begin{array}{l}\text { Position } \\
\text { (inches) }\end{array}$ & $\mathbf{0 . 0}$ & $\mathbf{1 . 0}$ \\
\hline $\mathbf{7 . 0}$ & $<1.7$ & $<0.8$ \\
\hline $\mathbf{6 . 5}$ & $<3.8$ & $<2.1$ \\
\hline $\mathbf{6 . 0}$ & $<4.3$ & $<6.1$
\end{tabular}


Table 227b.U. Uranium concentration $\left(\mathrm{mg} / \mathrm{cm}^{2}\right)$ as a function of position.

\begin{tabular}{l|l|l}
\hline $\begin{array}{l}\text { Position } \\
\text { (inches) }\end{array}$ & $\mathbf{0 . 0}$ & 1.0 \\
\hline $\mathbf{7 . 0}$ & $<7.2$ & $<9.0$ \\
\hline $\mathbf{6 . 5}$ & $<3.0$ & $<2.1$ \\
\hline $\mathbf{6 . 0}$ & $<2.0$ & $<0.6$
\end{tabular}

\section{CONCLUSIONS}

The X-ray K-edge detector proved very capable of quantifying heavy metal deposits in these drain lines. The results are in qualitative agreement with radiation survey measurements. It will not be possible to obtain quantitative verification without removing the pipes. The $\mathrm{K}$-edge measurements provided very good spatial resolution to define the limits of contamination. Deposits of mercury were also identified, a task that is not possible with most other non-invasive inspection techniques. This information should prove valuable in deciding how to remove these pipes. Decisions on where to cut the pipes can be made so as to minimize the potential for contaminating the surroundings.

One drawback of the K-edge system is the time required to complete the measurements. To get a complete picture of the contamination it is necessary to make measurements at different locations on the pipe. The deposits in these drain traps tended to be very inhomogeneous making it difficult to interpolate between measurements.

A total time of about one day was required to inspect each drain line. This included a setup time of 2-3 hours for each new room. A large part of this time was spent in assuring the radiation safety of the X-ray system. As these measurements were made in a building that was being used by the public for other activities, it was imperative that radiation levels be monitored and minimized in all areas around the $\mathrm{K}$-edge system where people could potentially be exposed. This necessitated placing shielding over some windows and restricting access to some adjoining rooms during operation of the X-ray system. This setup time could be greatly reduced for situations where a larger "exclusion zone" could be established around the X-ray tube. Long control cables allow the operator to sit up to 80 feet from the $\mathrm{X}$-ray tube.

Another factor was the time required to manually position the system for each measurement point on a drain line. This time could be reduced by automating the positioning of the source and detector. Another option that is being incorporated into a new version of the $\mathrm{K}$-edge detector is a real-time $\mathrm{X}$-ray imaging screen. This will allow rapid imaging of deposits and enable more accurate positioning of the Ge detector on regions of interest. 


\section{ACKNOWLEDGEMENTS}

We express our thanks to the many people at Ames Laboratory who assisted us in carrying out this demonstration. We are especially grateful to Tom Wessels and his staff in the Environment, Safety, Health, and Assurance Office for supporting these activities. This work has been supported under the DOE Characterization, Monitoring and Sensor Technology Crosscutting Program. The activities reported in this manuscript were funded by the Department of Energy through the Environmental Technology Development Program at Ames Laboratory, which is operated for the U.S. Department of Energy by Iowa State University under contract No. W-7405-ENG-82.

\section{REFERENCES}

1. "An Assessment of the Causes, Mitigation Efforts, and Current Status of ${ }^{232} \mathrm{Th},{ }^{238} \mathrm{U}$, and Beryllium Contamination in Wilhelm Hall at Ames Laboratory", Bruce W. Hokel, James H. Withers, W. Paul Waters, and Kenneth J. Ewing, Ames Laboratory Internal Report (1998).

2. "Field Demonstration of a Portable, X-Ray, K-Edge Heavy-Metal Detector",T.

Jensen, T. Aljundi, C. Whitmore, H. Zhong, and J.N. Gray, Ames Laboratory internal report IS-5131 (March 31, 1997).

3. "Application of X-ray K-Edge Densitometry in D\&D Operations", T. Jensen, T.

Aljundi, and J.N. Gray, Proceedings of the International Conference on Decommissioning and Decontamination and on Nuclear and Hazardous Waste Management, Spectrum '98, p. 902, The American Nuclear Society, La Grange Park, IL (1998). 


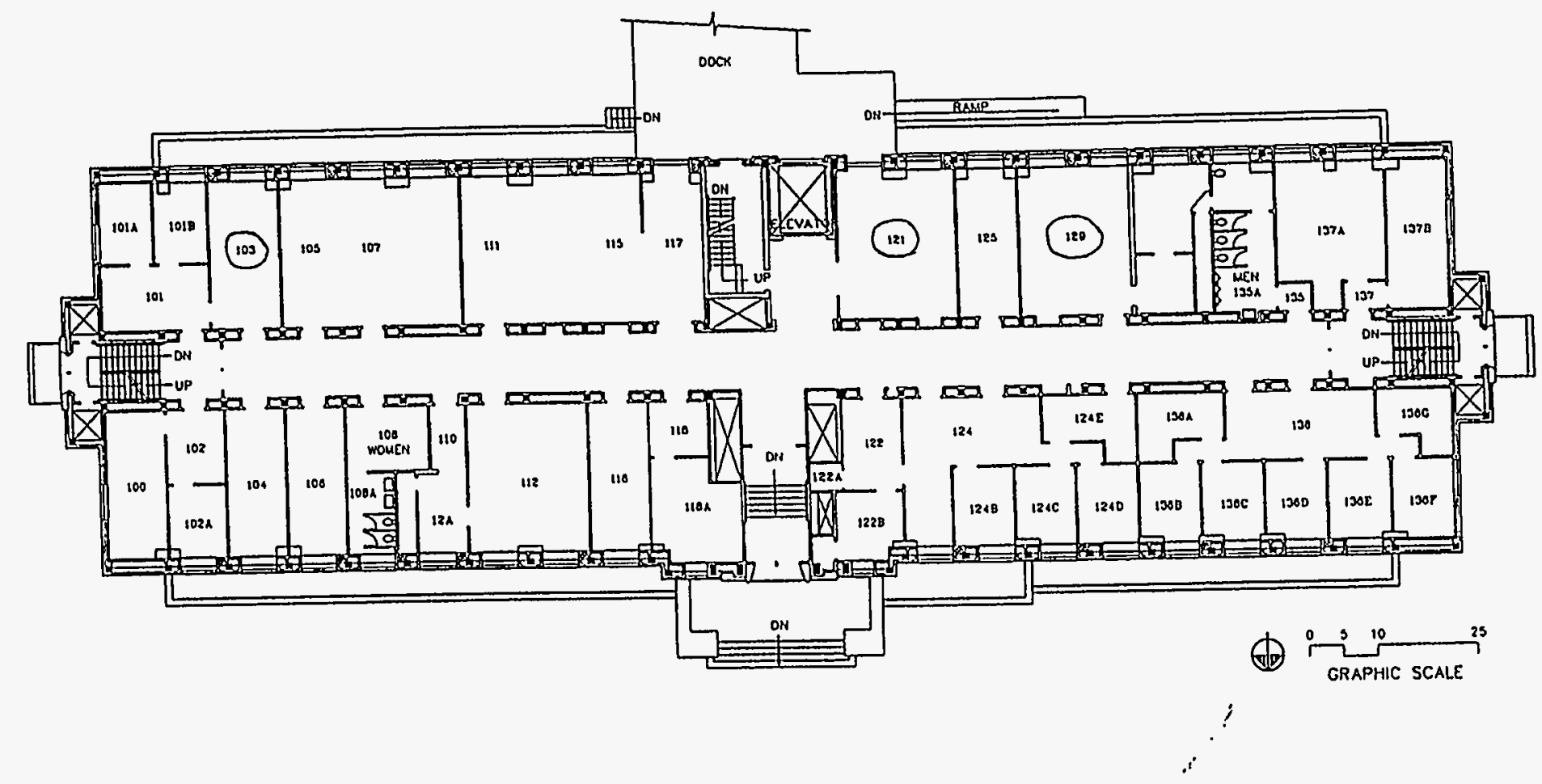



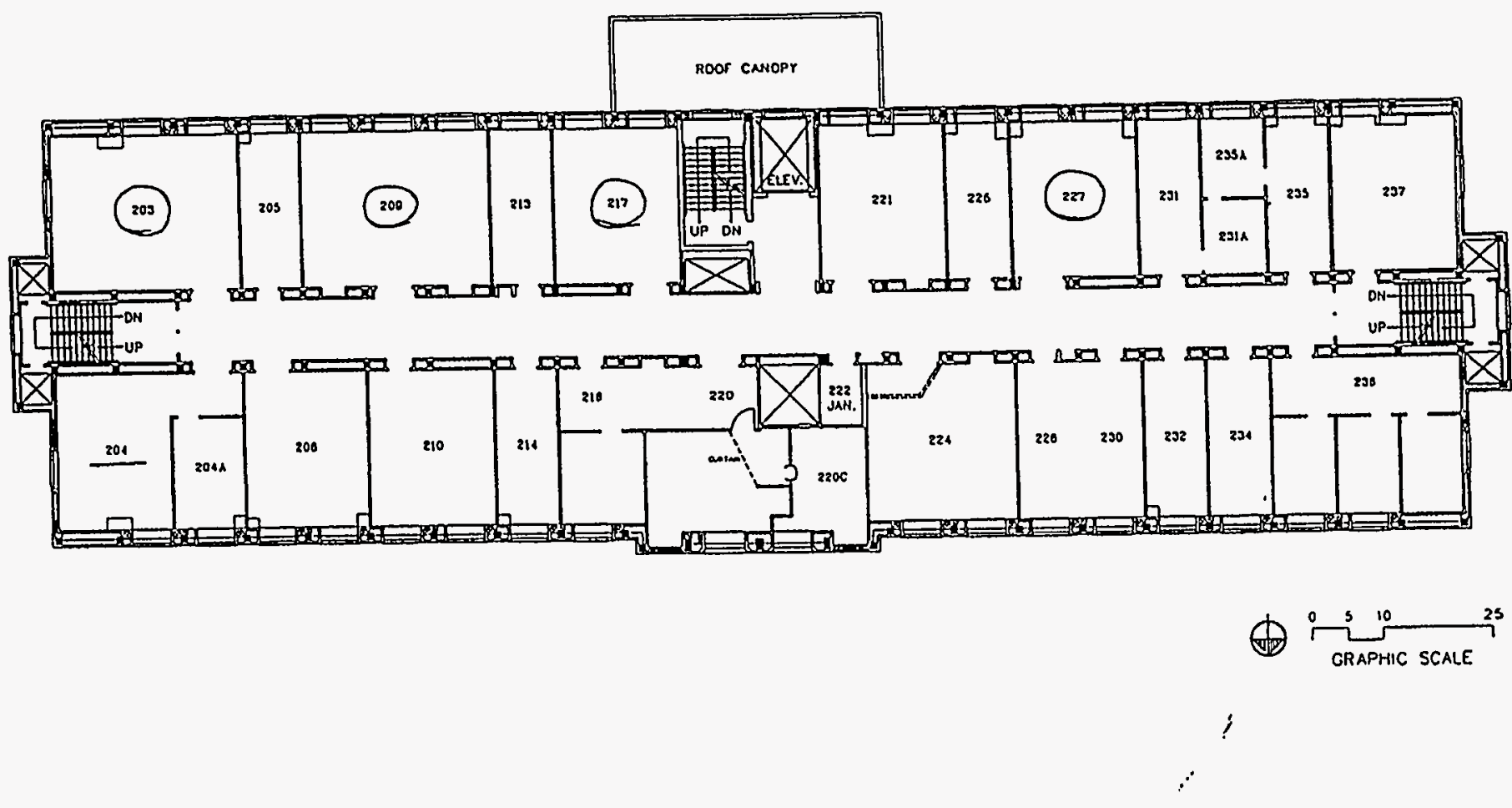
HWH_2 